\title{
COHERENT HARMONIC GENERATION EXPERIMENT ON UVSOR-II STORAGE RING
}

\author{
M. Labat, CEA, Saclay, France \\ G. Lambert, CEA, Saclay, France, RIKEN SPring-8 Harima, Hyogo, Japan \\ M.E. Couprie, Synchrotron Soleil, Saint-Aubin, France \\ D. Nutarelli, LAC, Orsay, France \\ M. Hosaka, A. Mochihashi, J. Yamazaki, M. Shimada, M. Katoh, IMS, Okazaki, Japan \\ Y. Takashima, Nagoya University, Nagoya, Japan \\ T. Hara, RIKEN SPring-8 Harima, Hyogo, Japan
}

\begin{abstract}
Harmonic Generation schemes on Free Electron Laser devices are very promising. The injection of a traditional laser source inside the first undulator of an optical klystron leads to an efficient energy modulation of the electron bunch, and therefore, its spatial modulation, resulting in a more coherent light emission along the second undulator. Experiments have been performed on the UVSOR-II Storage Ring at Okazaki (Japan) with electrons stored at an energy of $600 \mathrm{MeV}$, and using a $2.5 \mathrm{~mJ} \mathrm{Ti}$ :Sa laser at $800 \mathrm{~nm}$ wavelength, $1 \mathrm{kHz}$ repetition rate, and $150 \mathrm{fs}$ up to 2 ps pulse duration. The experimental setup is presented, including the transport alignment and synchronisation between the laser and the electron beam. We also report on the detection system, and the first results obtained.
\end{abstract}

\section{INTRODUCTION}

The Coherent Harmonic Generation (CHG) on storage rings $[1,2]$ results from the interaction between an intense seeded electric field, and a relativistic electron bunch. The seed can be either the own spontaneous emission of the electrons as demonstrated on DUKE [3] and ELETTRA [4] storage rings, or an external source [5] such as a high power laser. In this paper, we report successful generation of the third harmonic of a Ti:Sa laser on UVSOR-II storage ring.

The laser is focused in the first part of an optical klystron [6] (Modulator), and synchronized with the circulating electron bunch. The photon-electron beam interaction results in an energy modulation of the electrons, converted into density modulation after crossing a dispersive section. The electron beam is micro bunched at fundamental and sub harmonic wavelengths of the seeded laser. The light emission of the electrons is then enhanced in the second undulator (Radiator) at the third harmonic of the seed, i.e. $266 \mathrm{~nm}$. The general scheme is presented in Figure 1.

The seeded laser was initially optimized for Coherent Synchrotron Radiation experiments (CSR) [7] in the mm wavelength range on UVSOR-II, requiring high repetition rate $(1 \mathrm{kHz})$ and short pulse duration $(150 \mathrm{fs})$. Production from the same Ti:Sa laser of CSR and CHG is a performance, and will enable to deliver simultaneously a coher- ent, short duration and intense light in the far infra red as well as in the VUV range on UVSOR-II facility.

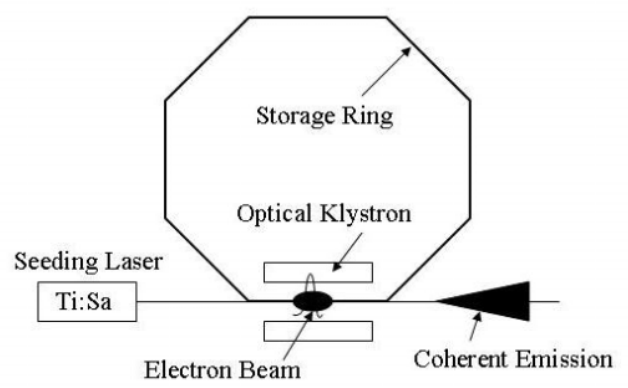

Figure 1: Coherent Harmonic Generation scheme on a storage ring

\section{ANALYTICAL MODEL FOR CHG}

Analytical calculation enabling the description of coherent harmonic emission in an optical klystron was developed by R. Coïsson and F. De Martini [8]. The intensity of the light emitted by a relativistic electron bunch in an optical klystron can be expressed as the sum of an incoherent and a coherent term. The coherent term equals zero for randomly distributed electronic phases. In the case of $\mathrm{CHG}$ process, and assuming that the energy modulation only occurs in the first undulator, the interaction between the electric field $E_{L}$ and the electrons induces an additional energy spread given by:

$$
\Delta \gamma=\frac{e K N E_{L} \lambda_{0}}{2 \gamma^{2} m c^{2}}\left(J_{0}(\xi)-J_{1}(\xi)\right),
$$

where $J$ are Bessel functions, depending of $\xi=$ $\frac{K^{2}}{4\left(1+K^{2} / 2\right)} . K$ is the undulator deflexion parameter, $\lambda_{0}$ the spatial period, $N$ the number of periods, $c$ the velocity of light, $e$ the electrons charge, $m$ their mass, and $\gamma$ their normalized energy. This energy spread results into an additional phase difference at the exit of the dispersive section, equal at its maximum to:

$$
\Delta \alpha=4 \pi\left(N+N_{d}\right) \Delta \gamma .
$$

$N_{d}$ is the number of equivalent periods of the dispersive section. The intensity of the coherent emission no longer 
averages to zero, and is given by the following expression:

$$
\frac{\delta W_{c o h}}{\delta w \delta \Omega}=\frac{n^{2} e^{2} N_{e}^{2} f_{n}^{2} J_{n}^{2}(n \Delta \alpha)}{16 \pi \epsilon_{0} c \lambda_{L}^{2}}\left(\frac{K N \lambda_{0}}{\gamma}\right)^{2} A_{n}^{2} .
$$

$n$ is the harmonic number, $N_{e}$ the number of electrons in the bunch, $f_{n}$ the modulation rate, $\lambda_{L}$ the seeded laser wavelength, and:

$$
A_{n}=1 / 2\left(J_{n+1}(n \xi)-J_{n-1}(n \xi)\right) .
$$

The intensity of the incoherent emission is given by:

$$
\frac{\delta W_{\text {incoh }}}{\delta w \delta \Omega}=\frac{n^{2} e^{2} N_{e}}{16 \pi \epsilon_{0} c \lambda_{L}^{2}}\left(1+f_{n}\right)\left(\frac{K N \lambda_{0}}{\gamma}\right)^{2} A_{n}^{2} .
$$

This model, already used for ACO [1] and Super-ACO [2] storage rings configurations, allows apprehending Coherent Harmonic Generation process and its dependency to electron beam and seeded laser parameters. In addition, calculations performed before the experimental sessions provided with reasonable expectations on the obtention of the third coherent harmonic with UVSOR-II storage ring, and its laser parameters.

\section{EXPERIMENTAL SETUP}

\section{Electron Beam and Seeded Laser}

In FEL operation, using single bunch mode, UVSOR-II [9] facility provides an electron beam at $600 \mathrm{MeV}$, with a natural energy spread of $0.034 \%$. Up to $40 \mathrm{~mA}$ of beam current $(I)$ can be stored in the ring without any beam instability. Because life time is rather short at high current, most of the experiments were performed between 1 and 10 $\mathrm{mA}$. The optical klystron [10] is made up of two identical undulators of 9 periods of $11 \mathrm{~cm}$, separated by a $33 \mathrm{~cm}$ long dispersive section.

The IR laser system installed at UVSOR-II is mainly composed of a mode-locked titanium-sapphire (Ti:Sa) laser oscillator (Coherent, Mira 900-F) and of a regenerative amplifier (Coherent, Legend HE), which delivers high intense femto-second pulses. The regenerative amplifier is driven by a $\mathrm{Q}$ switched pump laser. The typical characteristics of this laser are given in Table 1.

Table 1: Seeded Laser characteristics

\begin{tabular}{|l|c|c|}
\hline Parameter & Value & Unit \\
\hline Wavelength & 800 & $\mathrm{~nm}$ \\
Spectral width & 12 & $\mathrm{~nm}$ \\
Repetition rate & 1 & $\mathrm{kHz}$ \\
Pulse duration $(\Delta t)$ & 0.15 to 2 & $\mathrm{ps}$ \\
Average Power $\left(P_{L}\right)$ & 1.8 & $\mathrm{~W}$ \\
Diameter & 11.5 & $\mathrm{~mm}$ \\
Gaussian quality factor & 1.25 & \\
Polarization & Horizontal & \\
\hline
\end{tabular}

02 Synchrotron Light Sources and FELs

\section{Synchronisation}

In order to synchronise the laser pulse with the electron bunch which has to be heated, a timing system has been specially developed at UVSOR-II [11]. The modelocked Ti:Sa laser is synchronised with the RF signal ( $\left.f_{R F}=90.1 \mathrm{MHz}\right)$ of the storage ring. In addition, the repetition frequency $f_{Q}$ of the $\mathrm{Q}$ switched laser is based on the sub-harmonics of the revolution frequency of the bunches $f_{\text {rev }}=f_{R F} / 16=5.6 \mathrm{MHz}$, leading to $f_{Q}=f_{\text {rev }} / 5632=1 \mathrm{KHz}$. To select and fix the electron bunch with the laser pulse cuts, an RF bucket selector is used for making the Q switching trigger signal. The timing of the laser pulse within the bunch spacing time is adjusted using a phase shifter modifying the phase of the RF signal. The condition of synchronisation is observed using in first stage a photodiode, receiving both laser pulses and synchrotron radiation (SR). More accurate tuning is further performed using a streak camera, which temporal resolution reaches $10 \mathrm{ps}$.

\section{General layout}

Coherent Harmonic Generation process requires, in addition to synchronisation, spatial overlap, and therefore accurate alignment of the seeded laser on the electron trajectory in the optical klystron. The FEL oscillator at $800 \mathrm{~nm}$ is defining this axis. Auto collimation was then performed to mark the seeded laser path from the laser hutch through out the FEL cavity. The IR beam is transported by means of three periscopes, each using two flat mirrors at $45^{\circ}$ incidence, and then focused inside the modulator with a $5 \mathrm{~m}$ long focal lens in order to optimize the overlapping with the e-beam and increase the local energy. Both beams have horizontal polarization. The general layout of the optical set is shown in Figure 2.

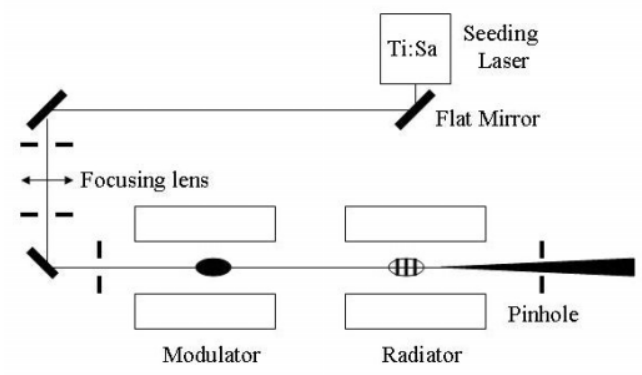

Figure 2: Setup of the laser transport to the FEL cavity.

\section{EXPERIMENTAL RESULTS}

The output radiation of the undulators is sent onto a solar blind PhotoMultiplier (PM, Hamamatsu, R759). An interferometric filter (CVI-F25-265) centered at $266 \mathrm{~nm}$ with $25 \mathrm{~nm}$ bandwidth enables the spectral selection of the third harmonic. In order to observe the coherent emission at 1 $\mathrm{KHz}$, among incoherent emission at $5.6 \mathrm{MHz}$, the PM signal is observed on an oscilloscope, triggered by the laser 
timing system. A picture of the oscilloscope screen is presented in Figure 3.

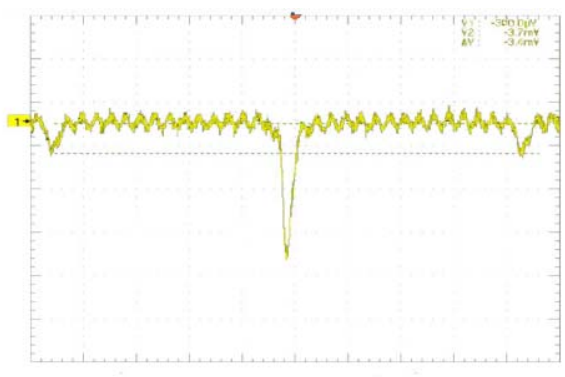

Figure 3: Screen capture of the oscilloscope, showing photomultiplier signal. Central peak corresponds to the sum of coherent emission and synchrotron radiation (incoherent emission), and edged peaks to synchrotron radiation. $P_{L}=1.78 \mathrm{~W}, \Delta t=1.12 \mathrm{ps}, I=4.29 \mathrm{~mA}$

Using the analytical model presented before, the CHG signal is expected to increase as the square of the number of electrons involved in the interaction, or the peak current since the electron bunch duration is much wider than the laser pulse duration (by two orders of magnitude). First results seem to confirm the expected quadratic behaviour.

Assuming a Gaussian distribution of the electron bunch, the electron-photon interaction term, and therefore the number of coherent photons emitted, will be maximized when the laser pulse hits the centre of the bunch. When delayed, the laser pulse shifts towards the edges of the electron bunch, where the electronic density falls back to zero. The CHG signal is then expected to disappear. The experimental results are in good agreement with those assumptions. Indeed, at $3 \mathrm{~mA}, \mathrm{CHG}$ signal can be detected over a variation of the delay of $360 \mathrm{ps,} \mathrm{with} \mathrm{total} \mathrm{annihilation}$ of CHG signal on the edges, which gives a width of $70 \mathrm{ps}$ (rms), to be compared with a 85 ps (rms) bunch length. Figure 4 presents an picture from Streak Camera, illustrating the optimised position for CHG of the laser pulse: in the centre of the electron bunch.

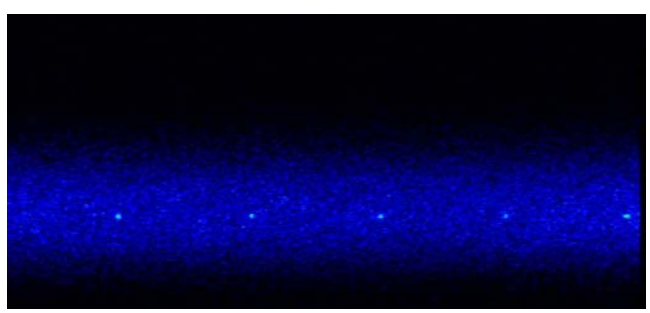

Figure 4: Streak Camera Image. Bright blue spots correspond to laser pulse, dark blue to SR. Full scales are $85 \mathrm{~ms}$ for the horizontal and 700 ps for the vertical axis.

$\mathrm{CHG}$ process is based on the amplification by the radiator of the sub harmonics of the seeding laser wavelength. To be properly amplified, this sub harmonic wavelength must correspond to a resonant wavelength of the optical klystron, defined by the undulator parameter $\mathrm{K}$, and therefore by its gap. Consequently, a detuning of this parameter might kill the radiation at $266 \mathrm{~nm}$. Indeed, $\mathrm{CHG}$ emission is maximum for an undulator gap of $40.8 \mathrm{~mm}$ and the signal vanishes for gaps of 39.7 and $42.5 \mathrm{~mm}$.

\section{CONCLUSION}

We have successfully observed for the first time the coherent harmonic generation from a $1 \mathrm{kHz} \mathrm{Ti}$ :Sa laser injected in an optical klystron installed on the UVSOR-II storage ring. The Ti:Sa laser specifications are well adapted for the CSR observations in UVSOR-II, but they also allow the CHG to be performed. Both constitute an important step for the seeded fourth generation light sources.

Further experiments foresee the measurement of the fifth harmonics, of the $\mathrm{CHG}$ effects on the electron beam, and the influence of relevant features such as seeded laser characteristics: energy, diameter, pulse duration, as well as shaping inside the modulator.

\section{ACKNOWLEDGEMENTS}

We would like to thank all the UVSOR-II staff for its help during experimental sessions. This work was supported by International Collaboration program of IMS.

\section{REFERENCES}

[1] R. Prazeres, J.M. Ortega, C. Bazin, M. Bergher, M. Billardon, M.E. Couprie, M. Velgue and Y. Petroff, Nucl. Inst. Meth. A 272 (1988) 68-72.

[2] R. Prazeres, P. Guyot-Sionnest, J. M. Ortega, D. Jaroszynski, M. Billardon, M. E. Couprie, M. Velghe and Y. Petroff, Nucl. Inst. Meth. A 304 (1991) 72-76.

[3] V. Litvinenko, Nucl. Inst. Meth. A 507, 265 (2003).

[4] G. De Ninno, M. B. Danailov, B. Diviacco, M. Ferianis and M. Trovo, FEL'04, August 2004, p. 237-240.

[5] P.L. Csonka, Part.accele. 8, 225 (1978).

[6] N.A. Vinokurov and A.N. Skrinsky, Preprint 77-59, Nuclear Physics Institute of Novossibirsk (1977).

[7] M. Katoh, M. Hosaka, S. Kimura, A. Mochihashi, M. Shimada, T. Takahashi, Y. Takashima and T. Hara, to be published in the Proceedings of EPAC'06, THPLS041.

[8] R. Coïsson, F. De Martini, Phys. of Quant. Electron., 9, p. 939, Addison-Wesley (1982).

[9] M. Katoh, M. Hosaka, A. Mochihashi, J. Yamazaki, K. Hayashi,Y. Hori,T. Honda, K. Haga, Y. Takashima, T. Koseki, S. Koda, H. Kitamura, T. Hara, T. Tanaka, AIP'04, 2004, (708) p. 49-52.

[10] H. Hama, K. Kimura, M. Hosaka, J. Yamazaki and T. Kinishita, Nucl. Instr. and Meth. A393 (1997) 23.

[11] A. Mochihashi, M. Hosaka, M. Katoh and Y. Takashima, UVSOR ACTIVITY REPORT 2005 (2006). 